\title{
Influence of Instructional Design to Manage Intrinsic Cognitive Load on Learning Effectiveness
}

\author{
Yi-Hung Huang ${ }^{1 *}$ \\ ${ }^{1}$ National Taichung University of Education, Taichung, TAIWAN \\ Received 7 April 2017 - Revised 24 January 2018 - Accepted 13 March 2018
}

\begin{abstract}
For elementary school students who are accustomed to solving single-layer mathematical problems, integrating multiple mathematical concepts and applying them to seek solutions is clearly a complex task. From the perspective of cognitive load, students may often find their intrinsic cognitive load exceeding their processing capacity when they face such tasks. This study investigated two strategies to manage intrinsic cognitive load: pretraining and segmented learning. The researcher first employed cognitive load theory to analyze the cognitive load faced by students using the two types of materials and then performed an experiment to verify theoretical analysis. At the same time, the researcher used the multidimensional cognitive load scale to gauge the cognitive loads perceived by the students. Experiment results showed that multidimensional cognitive load scale successfully measured the intrinsic load and extraneous load perceived by the students and pretraining resulted in lower perceived cognitive load than segmented learning. They further indicated that interaction effects exist between the two strategy groups and the high and low prior knowledge groups in posttest scores. This study suggests that pretraining is probably a more suitable instructional strategy to manage intrinsic load for most older elementary school students.
\end{abstract}

Keywords: cognitive load theory, intrinsic cognitive load, instructional design, mathematics education

\section{INTRODUCTION}

A comprehensive review of sixth-grade mathematics textbooks show that most units require the students to integrate the basic mathematics skills that they have acquired from first grade to fifth grade to solve problems in everyday life. Examples of these units include "How to Solve It" and "Reference Quantities and Comparison Quantities" (Huang, 2012). For elementary school students that are accustomed to solving problems using a single skill or concept, integrating multiple mathematical concepts and problem-solving procedures and then applying them is clearly a complex learning task. Indeed, students often find their intrinsic cognitive load exceeds their processing capacity when they face such tasks. Thus, the means of planning lessons appropriately to control intrinsic load during the instructional process and successfully achieve instructional objectives is crucial for sixthgrade mathematics teachers.

Cognitive load theory (CLT) posits that controlling intrinsic load depends on two aspects (Sweller, Ayres, \& Kalyuga, 2011). The first aspect, referred to as pretraining, targets the student's personal capabilities, wherein the basic schemata necessary to complete a new task is presented before combining multiple lower-level interactive elements into a single higher-level interactive element for processing. In this manner, intrinsic load associated with the learning process can be effectively reduced. Exemplar strategies of this approach include the pretraining principle presented by Mayer and Moreno (2010), the isolated elements effect proposed by Sweller et al. (2011), and the part-whole approach proposed by Kester, Paas, and van Merriënboer (2010). The second aspect, referred to as segmenting, targets the task itself; i.e., the order in which the task is presented. At first students are asked to process

(C) 2018 by the authors; licensee Modestum Ltd., UK. This article is an open access article distributed under the terms and conditions of the Creative Commons Attribution License (http://creativecommons.org/licenses/by/4.0/). $\square$ ehhwang@mail.ntcu.edu.tw (*Correspondence) 


\section{Contribution of this paper to the literature}

- The researcher designed systematically two sets of materials to manage intrinsic cognitive load to provide elementary school teachers with useful reference.

- The researcher compared representative instructional strategies in the two different aspects (pretraining and segmenting), with regard to their influence on perceived cognitive load and learning effectiveness.

- The researcher referred to the multidimensional cognitive load scale and successfully measured the intrinsic load and extraneous load perceived by the students.

subtasks with a smaller number of elements before the number of elements and the interactivity of the task are gradually increased. Instructional strategies in this aspect include the segmenting principle proposed by Mayer and Moreno (2010), and the whole-part approach presented by Kester et al. (2010).

The studies above demonstrate that instructional strategies can effectively manage intrinsic load and thereby enhance the learning effectiveness of students. However, no study has compared the instructional strategies of these two aspects. The purpose of this study was therefore to compare representative instructional strategies in the two different aspects (pretraining and segmenting), with regard to their influence on perceived cognitive load and learning effectiveness.

This study used the "Reference Quantities and Comparison Quantities" unit in sixth-grade mathematics curriculum in Taiwan to compare two strategies for intrinsic cognitive load management: pretraining and segmenting. First, the researcher designed two sets of learning materials based on the two instructional strategies and then employed CLT to analyze the cognitive load engendered by these materials. An experiment was then performed to examine the influence of the two instructional strategies on the learning effectiveness and cognitive loads of students with different levels of prior knowledge. In addition, the researcher used the multidimensional cognitive load scale developed by Leppink, Paas, van der Vleuten, Van Gog, and Van Merrienboer (2013) to gauge the cognitive load perceived by the students, assess the effectiveness of the multidimensional indicator, and thereby verify the results of theoretical analysis.

\section{LITERATURE REVIEW}

\section{Cognitive Load Theory}

CLT was proposed by Australian scholar Sweller (1988) to examine the influence of instructional design and learning content on learning and cognition. The structure of human cognition includes working memory, which processes conscious activities with a limited capacity, and long-term memory, which stores schema structures at different levels of automation with unlimited capacity. When the amount of information that an individual is processing exceeds the capacity or temporal limits of his or her working memory, it creates a cognitive load that interferes with the individual's cognitive process, which hinders learning.

In Sweller's (2010a) later revisions of the theory, he simplified the various sources of cognitive load into one, namely, element interactivity, and revised the types of cognitive load that an individual may perceive to include only intrinsic and extraneous cognitive load. This revised perspective enhanced the integrity of CLT. Intrinsic cognitive load is determined by the complexity of the learning material. The degree of intrinsic cognitive load depends on the interaction of the learner with the learning material. Extraneous cognitive load refers to the number of interactive elements included in the learning material or teaching process. Thus, from the perspective of CLT, the objective of instructional design is to reduce extraneous cognitive load, optimize intrinsic load, ensure that the sum of intrinsic load and extraneous load does not exceed the cognitive capacity and limits of the learners, and increase the use of germane resources as much as possible (Ayres, 2013; Kalyuga, 2015; Leppink \& van den Heuvel, 2015).

\section{Intrinsic Load Management}

Numerous instructional strategies have been developed in recent years to reduce extraneous load (Sweller, 2010a). However, how should educators proceed when the extraneous load has been eliminated as much as possible, but the intrinsic load still exceeds the learner's cognitive capacity?

Intrinsic cognitive load is determined by the complexity of the learning task. It can only be reduced by expanding the knowledge of the learner or altering the nature of the learning task. Therefore, relevant research has tackled this issue from one of two aspects (Sweller et al., 2011). The first aspect starts from the learner's own capabilities by first constructing the basic schemata needed to complete the new tasks and then combining multiple lower-level interactive elements into a single higher-level interactive element for processing. In this manner, the 
intrinsic load during the learning process can be effectively reduced. In accordance with the work of Mayer and Moreno (2010), we call this method 'pretraining'. This approach is used to develop specific prior knowledge; i.e., prior to the presentation of key materials. Learners initially need only complete a portion of the tasks with lower element interactivity and do not encounter tasks with greater element interactivity until later in the learning phase (Kester et al., 2010). Mayer and Moreno (2003) stated that teaching about the parts of the system before teaching about how the system works enhances learning effectiveness. Kester, Kirschner, and van Merriënboer (2004a, 2004b, 2006) agreed that reducing element interactivity at first helps learners process complex concepts. Research conducted by Pollock, Chandler, and Sweller (2002) and Clarke, Ayres, and Sweller (2005) on mathematics learning tasks revealed that when low-ability learners study from materials with high element interactivity, initially presenting only a small portion of the elements and their interactivity and then gradually including the rest promotes better learning transfer. Ayres $(2006 a, 2013)$ established that low-ability learners benefit from reduced element interactivity in the beginning, whereas high-ability learners benefit from high element interactivity early on in the learning process.

The other aspect starts from the task itself. Mayer and Moreno (2010) call this approach 'segmenting', in which the number and interactivity of elements are shown to the learners early on in the learning process. However, learners are required to process only subtasks, which include fewer elements. The number of elements and degree of interactivity are gradually increased as the complexity of the tasks executed by students increases (Kester et al., 2010). Creating a learning environment that focuses on subtasks helps to reduce the degree of interactivity among elements, by narrowing the number of elements learners must consider at any given time; i.e., releasing them from the difficulty of considering all of the elements in the task simultaneously. They learn by segmenting (Mayer et al., 2010) or with worked examples and completion tasks (Renkl \& Atkinson, 2010). Worked examples attract the learners' attention to the correct problem-solving process so that they do not need to waste cognitive resources on tasks unrelated to the correct problem-solving steps. In this manner, they reduce the element interactivity required for processing during the learning process. Once the learners have completely understood the worked examples, they can then construct problem-solving schemata by practicing completion tasks. Many studies have used worked examples and completion tasks to enhance the learning effectiveness of low-ability learners.

The studies above demonstrate that the instructional strategies of these two aspects can manage intrinsic load to some degree and thereby enhance learning effectiveness. However, no study has compared the instructional strategies of these two aspects. This study therefore compared representative instructional strategies in the two different aspects with regard to their influence on perceived cognitive load and learning effectiveness.

\section{Measurement of Cognitive Load}

Based on the characteristics of extraneous and intrinsic load, cognitive load is a multidimensional indicator. It can be perceived by learners, and it is a quantity that may fluctuate during the learning process. Despite the many research appeals to measure cognitive load (Ayres, 2006b; DeLeeuw \& Mayer, 2008; Galy, Cariou, \& Mélan, 2012), it remains unclear how best to quantify the different types of cognitive load adopting multiple items to gauge various types of cognitive load can provide more accurate measurements than using a single item and distinguish different types of cognitive load more clearly (Leppink, Paas, van der Vleuten, Van Gog, \& Van Merrienboer, 2014). As a result, Leppink et al. (2014) and Leppink and van den Heuvel (2015) suggested using six questions to gauge the intrinsic and extraneous load that learners perceive. Germane cognitive load, however, can only be indirectly verified via learning effectiveness. In consideration of factors such as location and method of implementation, the researcher adopted the aforementioned tool to measure the intrinsic and extraneous load that learners perceive and regarded learning effectiveness as an auxiliary objective reference value.

Based on CLT and the analysis of relevant literature, the questions that this study sought to answer are as follows:

1. Do students with different levels of prior knowledge perceive significantly different levels of intrinsic and extraneous load when reading two different learning materials that control intrinsic load?

2. Do students with different levels of prior knowledge display significant differences in learning effectiveness when reading two different learning materials that control intrinsic load?

\section{METHODOLOGY}

\section{Segmenting and Pretraining Learning Materials}

Previous researchers (Lee, 2013; Huang \& Shie, 2016) have reported that the "Reference Quantities and Comparison Quantities" unit in sixth-grade mathematics texts in Taiwan imposes high cognitive load. This can be attributed to the fact that the students must be able to identify the reference quantity as well as the comparison 
1. Huang has 20 pieces of candy. Hsing has $\frac{3}{5}$ the candy Huang has. How many pieces of candy do Huang and Hsing have altogether?

Step 1: Express the relationship between the quantities of candy that Huang and Hsing have using a line segment diagram.

As Hsing has $\frac{3}{5}$ the candy Huang has, the candy that Huang has serves as the reference quantity. Drawn as a line segment diagram, Huang's quantity of candy has can be drawn as a line that is 1 unit in length, while the number of pieces of candy that Hsing has can be drawn as a line that is $\frac{3}{5}$ of the length of the line representing the number of pieces of candy that Huang has.

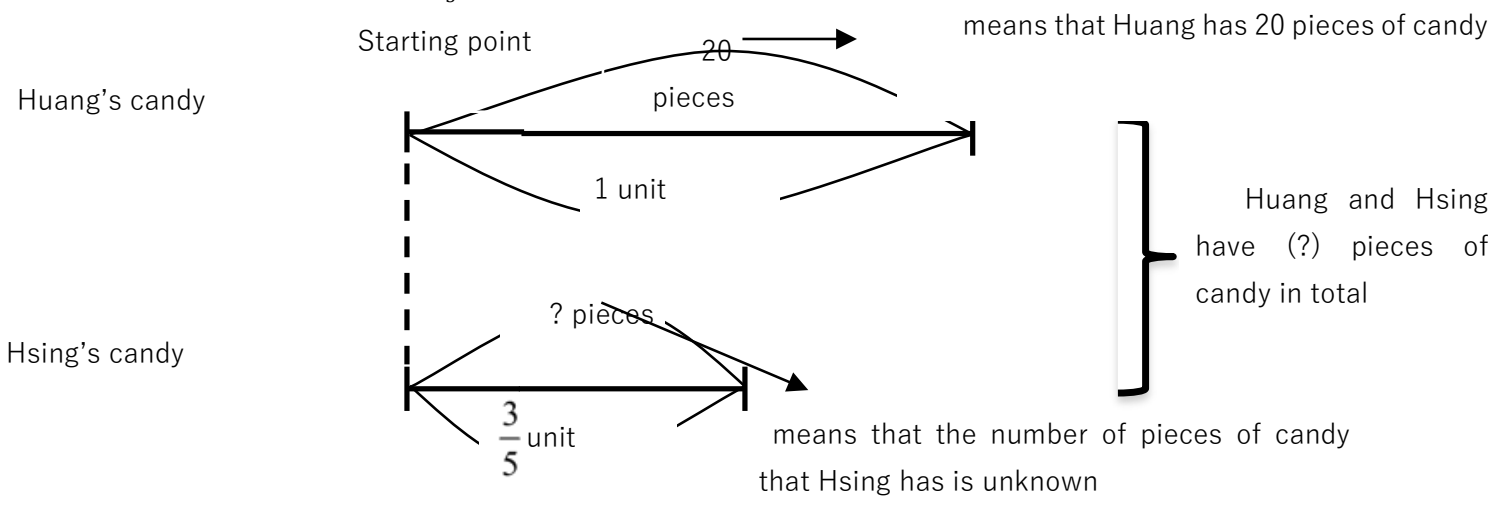

Step 2: Find the solution to the problem based on the line segment diagram.

The diagram above shows that Huang has 20 pieces of candy, whereas Hsing has an unknown quantity of candy. If the number of pieces of candy that Huang has is considered as a single unit, then the number of pieces of candy that Hsing has is $\frac{3}{5}$ of that unit.

Thus, Huang and Hsing have a total of $\left(1+\frac{3}{5}\right)$ units of candy.

Therefore, the number of pieces of candy that Huang and Hsing have in total is

$20 \times\left(1+\frac{3}{5}\right)=20 \times \frac{8}{5}=32$.

Ans: 32 pieces of candy in total

Figure 1. Worked example for segmented learning

quantity and adopt different problem-solving methods to solve the problems. The researcher therefore used this unit as the learning topic of the experiment in this study.

First, we present an example of segmented learning (Figure 1). The complete problem is shown at the beginning of the lesson. The first step is to explain how reference quantities and comparison quantities are to be identified, and the relationship between the two quantities are drawn in a line segment diagram. The second step is to explain how the clues in the line segment diagram are used to present the relationship in a mathematical expression. The students only need process a single step at a time, which reduces element interactivity and thereby manages intrinsic load.

When students finish reading a worked example, a similar example with less explanation is given in the form of a completion task, as shown in Figure 2. A blank space is left for the students to take notes in order that they may dedicate as much energy as possible to understanding the content, thereby easing germane load (Ayres, 2006a, 2013). The students are given the answer in the text; however, they must read the materials carefully to find it. This helps to ensure that they attend to the connection between $(1+4 / 5)$ and the equations, since the key point is to understand the concept on which the solving method is based. This instructional strategy starts from the task itself and arranges the subtasks in an appropriate order, from simpler tasks with lower element interactivity to the complete task with greater complexity and element interactivity. 
2. The teacher goes to the department store to buy a skirt and a pair of pants. The skirt is $\$ 200$, and the price of the pants is $\frac{4}{5}$ that of the skirt. How much did the teacher spend on her new clothes?

Step 1: Express the relationship between the prices of the skirt and the pants using a line segment diagram.

As the price of the pants is $\frac{4}{5}$ that of the skirt, the price of the pants serves as the reference quantity. Drawn as a line segment diagram, the price of the skirt can be drawn as a line that is 1 unit in length, while the price of the pants can be drawn as a line that is $\frac{4}{5}$ of the length of the line representing the price of the skirt.

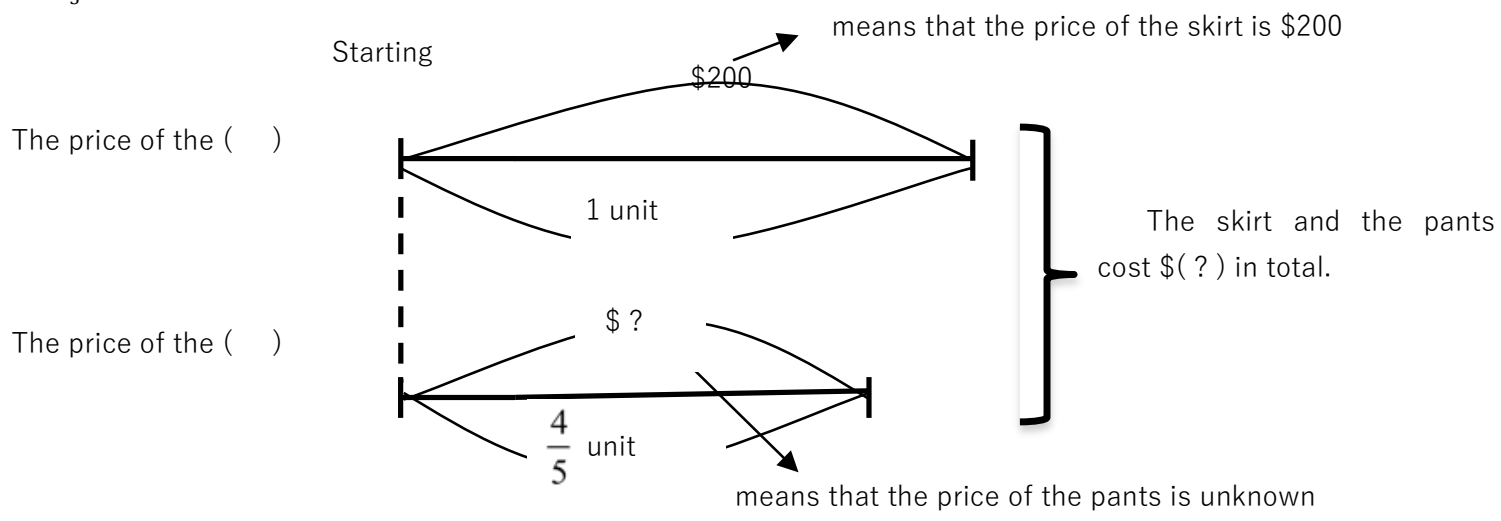

Step 2: Find the solution to the problem based on the line segment diagram.

The diagram above shows that the skirt is $\$ 200$, whereas the price of the pants is unknown. If the price of the skirt is considered 1 unit, then the price of the pants is $\frac{4}{5}$ unit.

Thus, the skirt and the pants cost ( ) units in total.

Therefore, the total cost of the skirt and the pants is $200 \times\left(1+\frac{4}{5}\right)=200 \times \frac{9}{5}=360$

Ans: They cost $\$ 360$ in total

Figure 2. Completion task for segmented learning

Next, we present the learning materials for the pretraining group. The example for this group is composed of two parts. The first part involves training the learners in the basic concepts needed to solve problems with reference quantities and comparison quantities, including identifying the reference quantity and drawing the line segment diagram. As with the segmented learning group, the students are given a similar problem to practice on after they have read a worked example, as shown in Figure 3. 
Training 1-1. Hsing has $\frac{3}{5}$ the candy that Huang has, which means that the quantity of candy that Huang has serves as the reference quantity. In a line segment diagram, the number of pieces of candy that Huang has can be drawn as a line that is 1 unit in length, while the number of pieces of candy that Hsing has can be drawn as a line that is $\frac{3}{5}$ of the length of the line representing Huang's candy.

Task: Use two parallel line segments to express the relationship between the candy that Huang and Hsing have.

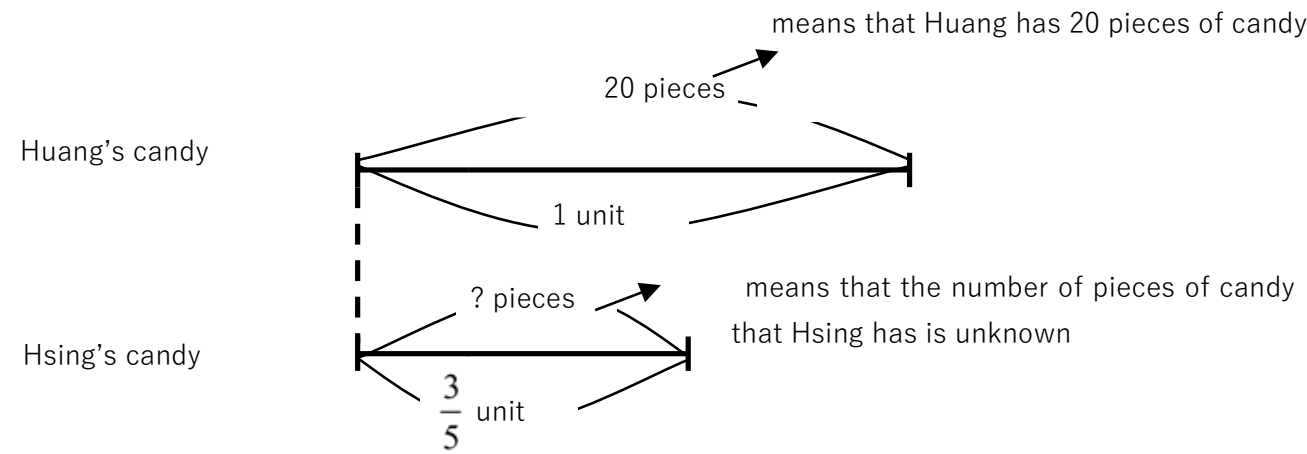

Training 1-2. The price of the pants is $\frac{4}{5}$ times that of the skirt. This means that the price of the pants serves as the reference quantity. In a line segment diagram, the price of the skirt can be drawn as a line that is 1 unit in length, while the price of the pants can be drawn as a line that is $\frac{4}{5}$ of the length of the line representing the price of the skirt.

Task: Use two parallel line segments to express the relationship between prices of the pants and the skirt.

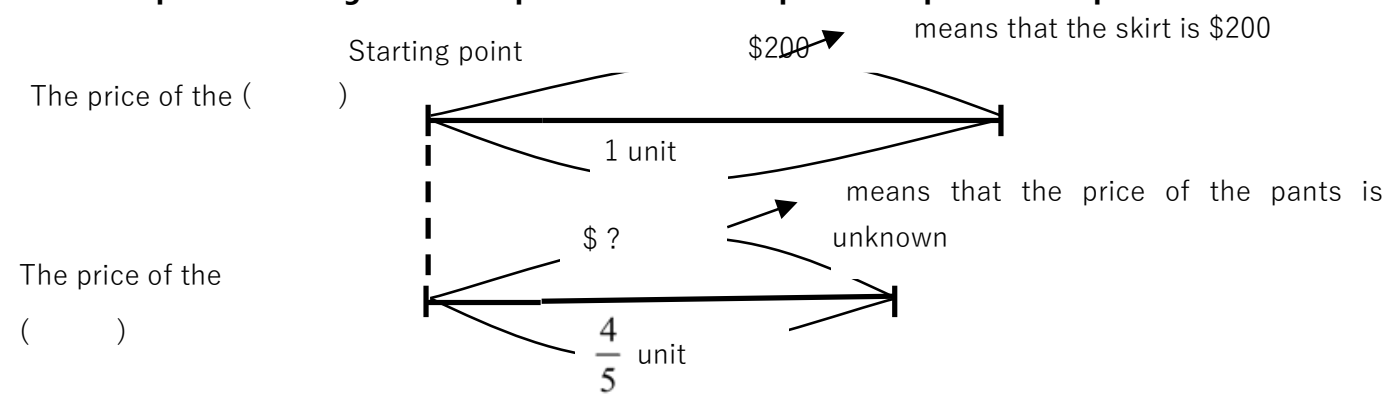

Figure 3. Worked example and complete task for drawing the line segment diagram

Next we present an explanation of how to translate the information held in a line segment diagram into a mathematical expression. This is followed by a similar problem on which students may practice. The materials are as shown in Figure 4 and Figure 5. 
Training 2-1. Use the line segments in the diagram below to find how many pieces of candy Huang and Hsing have altogether.

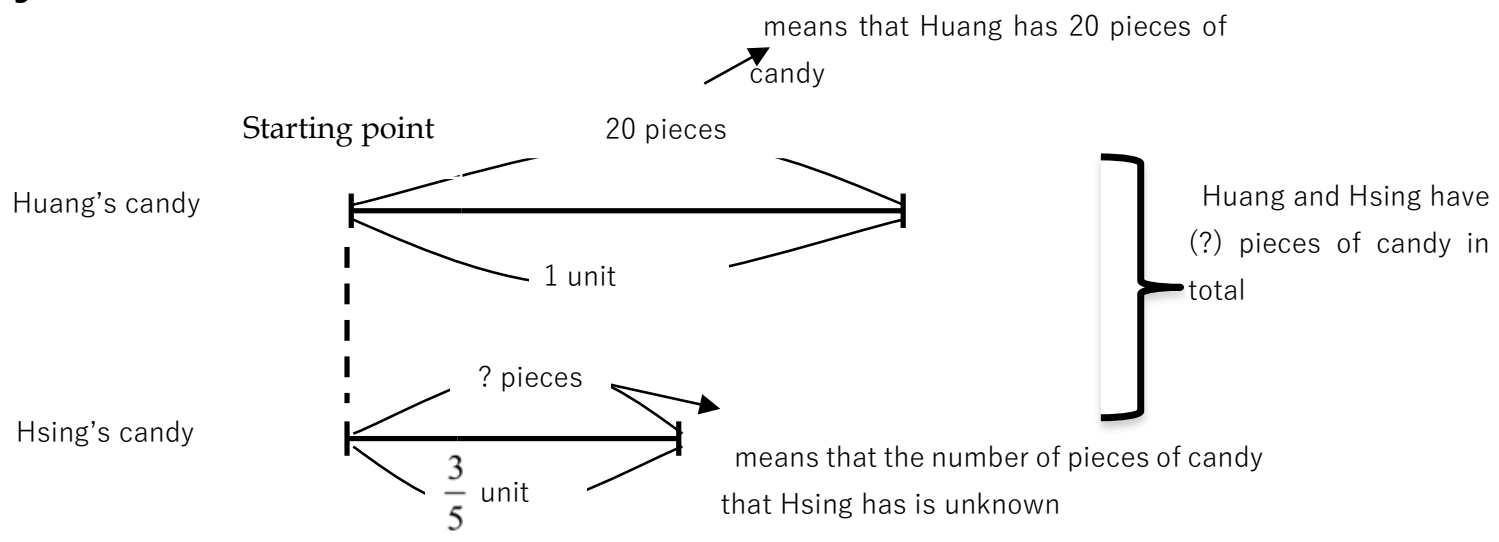

Task: Find the solution to the problem based on the line segment diagram.

The diagram above shows that Huang has 20 pieces of candy, whereas Hsing has an unknown number of pieces of candy. If the number of pieces of candy that Huang has is considered 1 unit, then the number of pieces of candy that Hsing has is $\frac{3}{5}$ of a unit. Thus, Huang and Hsing have a total of $\left(1+\frac{3}{5}\right)$ units of candy.

Therefore, the number of pieces of candy that Huang and Hsing have in total is

$$
20 \times\left(1+\frac{3}{5}\right)=20 \times \frac{8}{5}=32 \quad \text { Ans: } 32 \text { pieces of candy in total }
$$

Figure 4. Worked example for operating the line segment diagram

Training 2-2. Use the line segments in the diagram below to find how much the skirt and the pants cost in total.

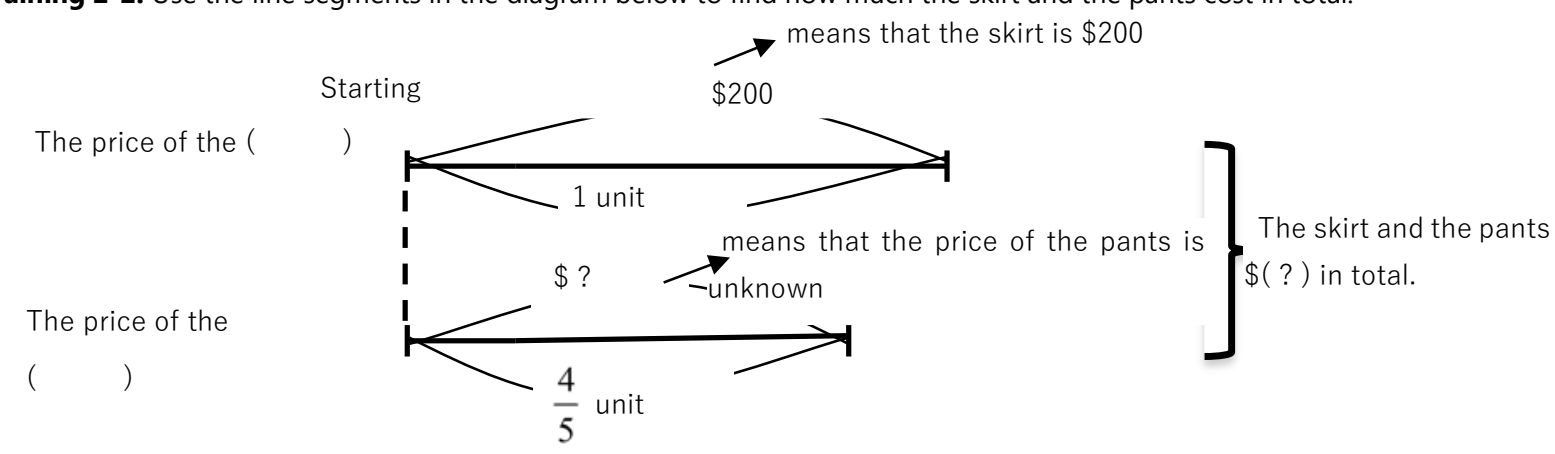

Task: Find the solution to the problem based on the line segment diagram.

The diagram above shows that the skirt is $\$ 200$, and the price of the pants is unknown. If the price of the skirt is considered 1 unit, then the price of the pants is $\frac{4}{5}$ of a unit. Thus, the skirt and the pants cost ( ) units in total.

Therefore, the total cost of the skirt and the pants is $200 \times\left(1+\frac{4}{5}\right)=200 \times \frac{9}{5}=360$

Ans: They cost $\$ 360$ in total

Figure 5. Completion task for operating the line segment diagram

The students do not learn how to solve a complete problem with reference quantities and comparison quantities until the second part. When the students finish reading a worked example, a similar completion task is provided for the students to practice on, as shown in Figure 6 and Figure 7. In the first part, they learn how to draw a line segment diagram to express the relationship between two objects and how to interpret the information held in a line segment diagram. Unlike the worked example for the segmented learning group, the two steps do not offer any instructional explanations; they merely present the line segment diagram representation and the mathematical expression. 
1. Huang has 20 pieces of candy. Hsing has $\frac{3}{5}$ the candy Huang has. How many pieces of candy do Huang and Hsing have altogether?

Step 1: Express the relationship between the numbers of pieces of candy that Huang and Hsing have:

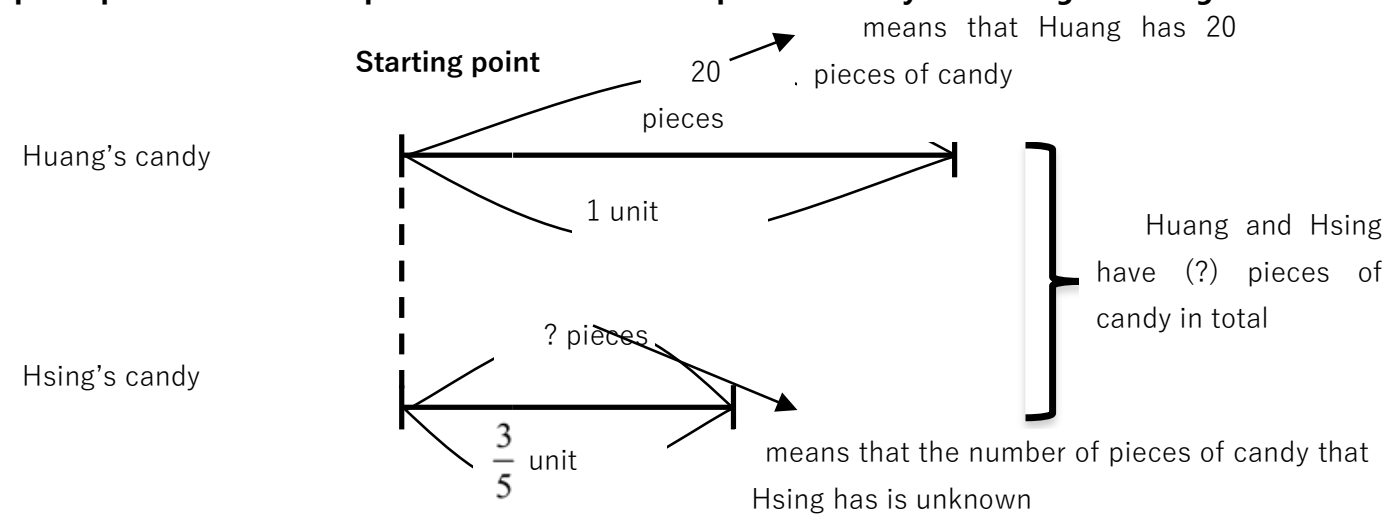

Step 2: Find the solution to the problem based on the line segment diagram.

The number of pieces of candy that Huang and Hsing have in total is

$20 \times\left(1+\frac{3}{5}\right)=20 \times \frac{8}{5}=32$

Ans: 32 pieces of candy in total

Figure 6. Worked example for pretraining group

2. The teacher goes to the department store to buy a skirt and a pair of pants. The skirt is $\$ 200$, and the price of the pants is $\frac{4}{5}$ that of the skirt. How much did the teacher spend on her new clothes?

Step 1: Express the relationship between the prices of the skirt and the pants using a line segment diagram.

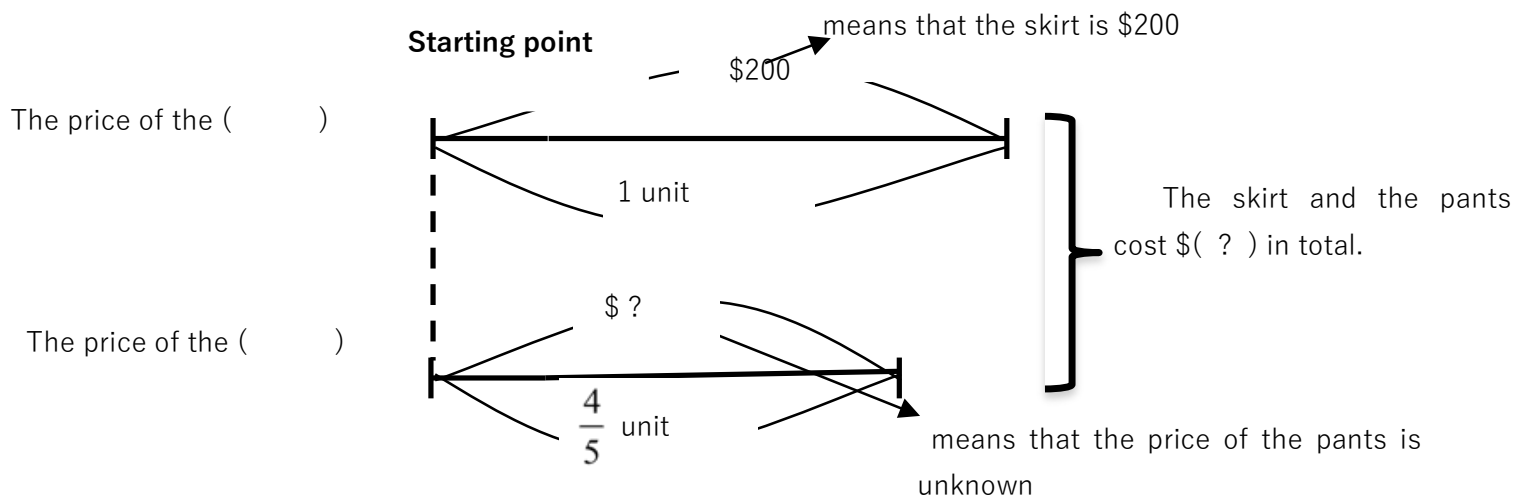

Step 2: Find the solution to the problem based on the line segment diagram.

Therefore, the total cost of the skirt and the pants is $200 \times\left(1+\frac{4}{5}\right)=200 \times \frac{9}{5}=360$

Ans: They cost $\$ 360$ in total

Figure 7. Completion task for pretraining group

The design principle of these learning materials is to first construct the basic schemata that the students need to complete the new task, namely, drawing the relationship between two objects using a line segment diagram and interpreting the line segment diagram representation. Once these basic schemata have been established, the students then learn how to use these two schemata to solve reference quantities and comparison quantities problems via the worked example. When students construct basic schemata, multiple interactive elements are merged into a single interactive element in process, which effectively reduces the intrinsic load during the learning process. 


\section{Research Hypotheses}

Existing research (Ayres, 2006a, 2013; Huang \& Shie, 2016) indicates that the prior knowledge influences a student's cognitive load and learning effectiveness. In view of this, the researcher investigated the cognitive load that learners with high and low prior knowledge may face during the learning process. Learners with high prior knowledge that can understand the concepts explained in Steps 1 and 2 in the example in Figure 1 need only focus on how to integrate the concepts to solve the problem. Segmented learning awakens their prior knowledge and assists the learner in constructing new problem-solving schemata. For learners with low prior knowledge, who are not as familiar with the relevant knowledge, pretraining enables learners to first construct the schemata of subconcepts, which contain fewer interactive elements and thus result in lower intrinsic load. Once they are familiar with the sub-concepts, the interactivity of the elements can be increased so that the leaners can construct the complete problem-solving schemata, which reduces their cognitive load.

Based on existing research (Ayres, 2006a, 2013) and the analysis above, the researcher inferred that segmented learning creates greater cognitive load than pretraining. Furthermore, prior knowledge interacts with the selected learning strategy. In other words, segmented learning is more effective than pretraining for students in the high prior knowledge group, whereas pretraining should be more effective than segmented learning for students in the low prior knowledge group. This difference is the result of the prior knowledge of the students.

\section{Participants}

The participants in the experiment of this study comprised 105 sixth-graders from four classes in an elementary school in Central Taiwan. The experiment was conducted one week after their midterms during the first semester. The curriculum used for the experiment was Unit 3 of Book 12 of the mathematics textbooks published by Nan-i Publishing Co. (Huang, 2012), which the students would have learned during the second semester. This prevented the possibility that students would have already come across the target content. To determine the influence of the two types of strategies on students with different levels of prior knowledge, we used the median of the students' midterm exam scores to divide the students into a high prior knowledge group and a low prior knowledge group. The students in these two groups were then randomly assigned to the segmented learning group and the pretraining group. The learning effectiveness and perceived cognitive load of the two groups were then compared. Using midterm scores to group the students had two merits. One is that the scope of the midterm exam covered division with fractions and ratios, which constitute the necessary prior knowledge for the "reference quantities and comparison quantities" unit. The students' midterm scores therefore served as an indicator of prior knowledge. The other merit was that a pretest, which would have affected the results of the posttest, was not needed to group the students.

\section{Experimental Design}

\section{Experimental procedure}

This experiment comprised two phases. During the first phase, the students read the materials to learn how to solve problems with reference quantities and comparison quantities. The second phase was the posttest, which assessed differences in learning effectiveness. Neither the pretraining group nor the segmented learning group were taught by a teacher; the learning examples were only presented on paper. The format of the problems and explanations in the examples were the same; i.e., they differed only in their presentation, as described above. This ensured that the students in the two groups experienced the same level of extraneous load but different levels of intrinsic load. During the learning process, the students were asked to fill out a cognitive load questionnaire after each pair of worked examples.

Phase 1: The students in the pretraining group first learned how to identify reference quantities and comparison quantities and draw line segment diagrams for the two pairs of corresponding examples. They were given three minutes to learn each pair of examples for a total of learning time of six minutes. Then, the students learned how to write mathematical expressions based on clues in the line segment diagrams. Again, they learned two pairs of corresponding examples. They were given three minutes of learning time for each pair of examples, so the learning time for this part was six minutes in total. Next, they learned how to solve two pairs of worked examples and completion tasks with reference quantities and comparison quantities. The reference quantity was unknown in one pair of problems, while the comparison quantity was unknown in the other pair of problems. They were given four minutes of learning time for each of the four problems, so the learning time for this part was 16 minutes in total. After a worked example and a completion task, the students were asked to fill out a cognitive load questionnaire. Each questionnaire took two minutes, so the two questionnaires took four minutes in total. Thus, the entire learning process for the pretraining group in Phase 1 was 32 minutes. 
1. The teacher bought a box of markers and a box of crayons at the stationary store. The box of markers was $\$ 72$, and the box of crayons cost $\frac{3}{4}$ as much as the box of markers. How much did the teacher spend in all?

2. John went to the supermarket to buy some fruit and vegetables. The vegetables cost $\$ 128$ in total, and the fruit cost $\frac{3}{4}$ as much as the vegetables. What was the difference between the cost of the vegetables and the cost of the fruit?

3. Lily planted some red and white roses in the garden. The white roses covered 144 hectares, and the white roses covered $\frac{3}{4}$ as much area as the red roses. What was the difference between the area of the white roses and the area of the red roses?

4. There are 84 yellow and blue marbles in total. There are $\frac{3}{4}$ times as many yellow marbles as there are blue marbles. How many yellow marbles are there? How many blue marbles are there?

5. Among the members of the choir this year, the number of girls is $\frac{2}{5}$ times that of the number of boys. The difference between the number of boys and the number of girls is 96 . How many boys are there? How many girls are there?

Figure 8. Posttest problems

In the segmented learning group, the students were given the complete problems at the beginning, following which learning tasks were given to teach each portion in segments. The students were given two pairs of worked examples and completion tasks with reference quantities and comparison quantities. The reference quantity was unknown in one pair of problems, while the comparison quantity was unknown in the other pair of problems. They were given seven minutes of learning time for each of the four problems, so the learning time for this part was 28 minutes in total. After a worked example and a completion task, the students were asked to fill out a cognitive load questionnaire. Each questionnaire took two minutes, so the two questionnaires took four minutes in total. Thus, the total learning time for the segmented learning group was also 32 minutes, thereby enabling the students in the two groups to learn under the same conditions.

Phase 2: After reading the materials, the two groups of students took a posttest containing five problems to determine the influence of the two different instructional strategies on their learning effectiveness and transfer of learning ability. The first three problems in the posttest involved near transfer of learning, whereas the last two problems involved far transfer of learning. The posttest scores were recorded in points.

The independent variables of this experiment were the prior knowledge of the students and the two different learning strategies, whereas the dependent variables were perceived cognitive load and learning achievement. The control variables were the learning content, the learning location, and paper learning materials.

\section{Pretraining materials}

The pretraining materials contained three parts. The first part focused on drawing line segment diagrams and contained two pairs of problems, each pair comprising an example and a problem-solving exercise, as shown in Figure 3. This part aimed to enhance student understanding of how to draw line segment diagrams based on the meaning of the problem. The second part focused on the interpretation of line segment diagrams. Again, this part contained two pairs of problems, each pair comprising an example and a problem-solving exercise, as shown in Figure 4 and Figure 5. This part aimed to enhance student understanding of mathematical expressions based on line segment diagrams. The third part contained the worked example and completion task, as shown in Figure 6 and Figure 7. The practice scores were recorded in points in the third part.

\section{Segmented learning materials}

The segmented learning materials display the problem at the beginning and then present the learning tasks of each segment. The learners need only focus on the individual task at hand, thereby reducing intrinsic load. Figures $\mathbf{1}$ and $\mathbf{2}$ display the examples in the segmented learning materials. The practice scores were recorded in points.

\section{Posttest}

The objective of the posttest is to gauge the effectiveness of near and far transfer of learning. The test contained five problems: The first three problems having a similar structure with the problems in the learning materials assessed the near transfer of learning, while the last two problems having a different structure with the problems in the learning materials assessed the far transfer of the students' learning. Figure $\mathbf{8}$ displays the problems used in the posttest. Each problem counted for two points, one for each of the two steps in the learning example.

\section{Cognitive load assessment questionnaire}

The questionnaire was designed as suggested by Leppink and van den Heuvel (2015). However, considering the fact that elementary students may not be able to differentiate between different levels, the researcher simplified 
Please answer the six questions below based on your feelings after reading the materials. Please choose one answer for each question.

Please read each question carefully, think about it, and then write down your answer.

( ) 1. The content of the materials that I just read were (1) not complicated at all (2) a little complicated (3) half complicated (4) mostly complicated (5) completely complicated.

( ) 2. The problems in the materials that I just read were (1) not complicated at all (2) a little complicated (3) half complicated (4) mostly complicated (5) all complicated.

( ) 3. Of the materials that I just read, (1) nothing was complicated (2) a few parts were complicated (3) half of the parts were complicated (4) most of the parts were complicated (5) all of the parts were complicated.

( ) 4. The explanations in the materials that I just read were (1) all very clear (2) a little unclear (3) half clear and half unclear (4) mostly unclear (5) all very unclear.

( ) 5. I understood (1) all (2) most (3) half (4) only a little (5) none of the explanations in the materials that I just read.

( ) 6 . I learned (1) all (2) most (3) half (4) only a little (5) none of the materials that I just read.

Figure 9. Cognitive load scale

Table 1. Descriptive statistics of learning effectiveness of various groups

\begin{tabular}{|c|c|c|c|c|c|c|}
\hline \multirow{2}{*}{$\begin{array}{l}\text { Instructional } \\
\text { strategy group (Full } \\
\text { score) } \\
\text { Prior knowledge } \\
\text { group } \\
\end{array}$} & \multicolumn{2}{|c|}{ Pretraining M (SD) } & \multicolumn{2}{|c|}{ Segmented learning $M$ (SD) } & \multicolumn{2}{|c|}{ Subtotal M (SD) } \\
\hline & High & Low & High & Low & High & Low \\
\hline Number of students & 24 & 25 & 26 & 24 & 50 & 49 \\
\hline Posttest score (10) & $7.25(2.17)$ & $3.28(1.60)$ & $5.35(2.47)$ & $3.67(2.26)$ & $6.26(2.50)$ & $3.47(1.94)$ \\
\hline Subtotal & \multicolumn{2}{|c|}{$5.22(2.75)$} & \multicolumn{2}{|c|}{$4.54(2.49)$} & & \\
\hline Practice score (5) & $4.96(.20)$ & $4.92(.27)$ & $4.69(.74)$ & $4.33(.82)$ & $4.82(.56)$ & $4.63(.67)$ \\
\hline Subtotal & \multicolumn{2}{|c|}{$4.94(.24)$} & \multicolumn{2}{|c|}{$4.52(.79)$} & & \\
\hline Intrinsic load (5) & $1.13(0.22)$ & $1.39(0.82)$ & $1.35(0.49)$ & $1.67(0.65)$ & $1.24(0.39)$ & $1.52(0.75)$ \\
\hline Subtotal & \multicolumn{2}{|c|}{$1.26(0.61)$} & \multicolumn{2}{|c|}{$1.50(0.59)$} & & \\
\hline Extraneous load (5) & $1.19(0.61)$ & $1.37(0.66)$ & $1.16(0.36)$ & $1.59(0.59)$ & $1.17(0.49)$ & $1.48(0.63)$ \\
\hline Subtotal & \multicolumn{2}{|c|}{$1.28(0.64)$} & \multicolumn{2}{|c|}{$1.37(0.53)$} & & \\
\hline
\end{tabular}

the nine-point scale to a five-point scale. The questions were as shown in Figure 9. The first three questions served as the indicator for intrinsic load, while the last three problems served as the indicator for extraneous load.

\section{Data analysis}

The data analysis in this study was conducted using SPSS for MS Windows 18.0. Using factor analysis, the results of the first three questions in the cognitive load scale were extracted as intrinsic load and the results of the last three questions as extraneous load. With the high and low prior knowledge group and the two different learning groups as the independent variables and the posttest scores and cognitive load indicators as the dependent variables, a two-factor analysis of variance was conducted to determine the influence of the prior knowledge grouping and instructional design grouping on perceived cognitive load and posttest scores.

\section{RESULTS}

\section{Descriptive Statistics}

After eliminating six students with incomplete tests, the researcher analyzed the data from the remaining 99 students, among which 49 were in the pretraining group and 50 were in the segmented learning group. The descriptive statistics of the high and low prior knowledge groups with regard to posttest scores, practice scores, intrinsic load, and extraneous load are presented in Table 1. 


\section{Results}

\section{Cognitive load indicator analysis}

Factor analysis of the intrinsic load confirmed the scale of measurement with an eigenvalue of 2.71, which accounted for $90.33 \%$ of the total variance. Factor analysis of the extraneous load confirmed the scale of measurement with an eigenvalue of 2.56 , which accounted for $85.38 \%$ of the total variance. The internal consistency of our questionnaires was satisfactory for both intrinsic load (Cronbach's alpha $=.95$ ) and extraneous load (Cronbach's alpha $=.91$ ).

Based on the experiment results, the research performed a two-factor analysis of variance on the measurements of intrinsic load in the two instructional strategy groups and high and low prior knowledge groups. Levene's test for homogeneity was first conducted. The intrinsic load result was $F(3,95)=2.549, p=.060$, which does not reach significance. This indicates homogeneity and that the analysis of variance can be performed. The interaction effects between the two strategy groups and high and low prior knowledge groups resulted in $F(3,95)=.077, p=.783$, which does not reach significance. Thus, no interaction effects existed between the two strategy groups and high and low prior knowledge groups. As the interaction effects were not statistically significant, the main effects of the two strategy groups and high and low prior knowledge groups were examined.

The analysis result for the two strategy groups in intrinsic load was $F(3,95)=4.333, p=.040$, which reaches the level of significance. Thus, the mean of intrinsic load of the pretraining group, 1.26, is significantly lower than that of the segmented learning group, 1.50. This result verifies the previous inferences in that the pretraining strategy will result in lower perceived cognitive load than the segmented learning strategy. The analysis result for the high and low prior knowledge groups in intrinsic load was $F(3,95)=5.899, p=.017$, which reaches the level of significance. Thus, the mean of intrinsic load of the high prior knowledge group, 1.24, is significantly lower than that of the low prior knowledge group, 1.52.

A two-factor analysis of variance was then performed on the measurements of extraneous load in the two instructional strategy groups and high and low prior knowledge groups. Levene's test for homogeneity was first conducted. For extraneous load, $F(3,95)=1.662, p=.180$, which does not reach significance. This indicates homogeneity and that the analysis of variance can be performed. The interaction effects between the two strategy groups and high and low prior knowledge groups resulted in $F(3,95)=.1 .212, p=.274$, which does not reach significance. Thus, no interaction effects existed between the two strategy groups and high and low prior knowledge groups. As the interaction effects were not statistically significant, the main effects of the two strategy groups and high and low prior knowledge groups were examined.

The analysis result for the two strategy groups in extraneous load was $F(3,95)=.749, p=.389$, which does not reach the level of significance. This means that the pretraining group and the segmented learning group displayed no significant differences in extraneous load. The analysis result for the high and low prior knowledge groups in extraneous load was $F(3,95)=7.187, p=.009$, which reaches the level of significance. Thus, the mean of extraneous load of the high prior knowledge group, 1.17, is significantly lower than that of the low prior knowledge group, 1.48 .

\section{Posttest score analysis}

To determine whether students with different levels of prior knowledge displayed differences in their posttest scores in the pertaining group and the segmented learning group, a two-factor analysis of variance was conducted with the posttest scores as the dependent variable and the high and low prior knowledge groups and the two strategy groups as the independent variables. Levene's test for homogeneity was first conducted. For the posttest scores, $F(3,95)=1.512, p=.216$, which does not reach significance. This indicates homogeneity and that the analysis of variance can be performed. The result of interaction effects between the two strategy groups and high and low prior knowledge groups was $F(3,95)=7.021, p=.009$, which reaches significance. Thus, significant interaction effects existed between the two strategy groups and high and low prior knowledge groups. As the interaction effects were statistically significant, the simple main effects of the two strategy groups and high and low prior knowledge groups were examined.

The prior knowledge groups were processed first. In the high prior knowledge group, the posttest score result was $F(1,48)=8.339, p=.006$, which indicates that the means of posttest score of the pretraining group, 7.25 , is significantly higher than that of the segmented learning group, 5.35. In the low prior knowledge group, the posttest score result was $F(1,47)=.482, p=.491$, which indicates no significant difference between the means of posttest score of the pretraining group, 3.28, and that of the segmented learning group, 3.67.

Next, the strategy groups were processed. In the pretraining group, the posttest score result was $F(1,47)=$ $53.501, p=.000$, which indicates that the means of posttest score of the high prior knowledge group, 7.25 , is 
significantly higher than that of the low prior knowledge group, 3.28. In the segmented learning group, the posttest score result was $F(1,48)=6.276, p=.016$ which means that the means of posttest score of the high prior knowledge group, 5.35, is significantly higher than that of the low prior knowledge group, 3.67.

\section{DISCUSSION}

\section{Analysis of Research Conclusions}

This study compared two strategies to manage intrinsic cognitive load, pretraining and segmented learning, using the unit Reference Quantities and Comparison Quantities in the sixth-grade mathematics curriculum in Taiwan. First, the researcher designed two sets of materials based on the two instructional strategies and then employed CLT to analyze the possible cognitive load that learners may face when using the two types of materials. Next, the researcher performed an experiment on learners with different levels of prior knowledge to investigate the influence of the instructional strategies on learning effectiveness and perceived cognitive load. At the same time, the multidimensional cognitive load scale developed by Leppink et al. (2013) was employed to gauge the cognitive loads perceived by the learners, assess the effectiveness of the multidimensional indicator, and thereby verify the results of our theoretical analysis. The researcher thereby arrived at the following conclusions.

\section{Pretraining results in lower cognitive load than segmented learning}

Existing CLT research on elementary school students used conventional single items as indicators of the cognitive load perceived by the students (Huang \& Shie, 2016; Wong, Leahy, Marcus, \& Sweller, 2012). However, misunderstanding of questionnaire items or insufficient sensitivity with regard to the indicators can lead to errors in the measurement of cognitive load. To overcome this, this study referred to the multidimensional cognitive load scale developed by Leppink et al. (2013) to measure the intrinsic and extraneous load perceived by the students. Use of this scale increased the validity of our finding that pretraining results in lower perceived cognitive load than segmented learning. This result is consistent with the researcher's hypothesis in that the different strategy groups displayed significant differences in intrinsic load but no significant differences in extraneous load. The results of the statistical analysis also verified the researcher's inference that pretraining results in significantly less intrinsic load. To further verify the accuracy of this analysis, the researcher also looked at how the students performed in the completion tasks while reading the materials. As the students had similar examples to refer to when they did the completion tasks, most of them obtained full marks. The practice scores did not follow a normal distribution, so the researcher adopted a nonparametric test to determine whether significant differences existed between the two strategy groups in the practice scores. The results of a Mann-Whitney U test showed significant differences between the two strategy groups with $p=.000$. During the learning process, the segmented learning group displayed significantly greater perceived cognitive load than the pretraining group, which affected their performance in the completion tasks. As a result, the practice scores of the students in the segmented learning group were significantly lower than those of the students in the pretraining group.

Furthermore, in both groups, the students in the high prior knowledge group presented significantly lower intrinsic load and extraneous load than the students in the low prior knowledge group. This also shows that prior knowledge indeed influences the intrinsic and extraneous load perceived by learners.

\section{Interaction effects between two strategy groups and high and low prior knowledge groups in posttest scores}

In the analysis of the posttest scores, interaction effects existed between the two strategy groups and the high and low prior knowledge groups, which means that prior knowledge indeed influences the instructional strategy adopted to manage intrinsic load. This is consistent with our inferences and the findings of previous research (Ayres, 2006a, 2013). However, statistical analysis revealed that among the students in the high prior knowledge group, those who experienced pretraining obtained significantly higher posttest scores than those in the segmented learning group, which is inconsistent with the researcher's hypothesis. The research speculates that this was because the students in the high prior knowledge group did not have sufficient prior knowledge to understand the segmented learning materials. The previous cognitive load indicator analysis revealed that the students in the pretraining group perceived lower cognitive load than those in the segmented learning group. The CLT emphasizes that when the cognitive load exceeds the learner's cognitive capacity, it affects his or her learning effectiveness. The researcher therefore infers that when the students in the high prior knowledge group were learning with the pretraining materials, the resulting cognitive load did not exceed their cognitive capacity. In contrast, the cognitive load resulting from the segmented learning materials exceeded the cognitive capacity of the high prior knowledge 
Table 2. Statistics of student performance in first three problems of posttest

\begin{tabular}{|c|c|c|c|c|c|c|}
\hline \multirow{2}{*}{\begin{tabular}{|l|} 
Instructional strategy group \\
Prior knowledge group
\end{tabular}} & \multicolumn{2}{|c|}{ Pretraining } & \multicolumn{2}{|c|}{ Segmented learning } & \multicolumn{2}{|c|}{ Subtotal } \\
\hline & High & Low & High & Low & High & Low \\
\hline Number of students & 24 & 25 & 26 & 24 & 50 & 49 \\
\hline Application of single method & 8 & 25 & 19 & 18 & 27 & 43 \\
\hline Subtotal & & & & & & \\
\hline Correct application of two methods & 12 & 0 & 6 & 4 & 18 & 4 \\
\hline Subtotal & & & & & & \\
\hline Incorrect application of two methods & 4 & 0 & 1 & 2 & 5 & 2 \\
\hline Subtotal & & & & & & \\
\hline
\end{tabular}

students in the segmented learning group. As a result, the high prior knowledge students in the pretraining group obtained significantly higher posttest scores than those in the segmented learning group.

The statistical analysis results of the low prior knowledge group also did not support previous inferences: among the students in the low prior knowledge group, those in the pretraining group did not obtain significantly higher posttest scores than those in the segmented learning group. The researcher speculates that although the cognitive load indicator revealed that the cognitive load perceived by the pretraining group was lower than that perceived by the segmented learning group, the cognitive load resulting from both sets of materials still exceeded the cognitive capacity of the students in the low prior knowledge group. This resulted in no significant differences between the two groups of low prior knowledge students in the posttest scores.

To further verify the inferences above regarding the posttest scores of the students, the researcher analyzed the answers given by the students on the posttest. As mentioned in the section on research instruments, the first three problems in the posttest involved near transfer of learning. Based on the posttest scores in Table 1, only the high prior knowledge students in the pretraining group obtained scores greater than 6 points, which means most of these students solved these three problems correctly. However, the mean score of high prior knowledge students in the segmented learning group did not reach 6 points, which means that even some of these students still could not solve all of the near transfer of learning problems correctly, let alone the low prior knowledge students in the two strategy groups, whose mean score was less than 6 points. This also indicates that the overall rate of answering the two far transfer of learning problems in the posttest was low. For this reason, the researcher only analyzed the performance of the students in the three near transfer of learning problems.

The first and third of these three problems involve an unknown comparison quantity, whereas the second problem involves an unknown reference quantity. The students must be able to identify which is the reference quantity and which is the comparison quantity and adopt two different problem-solving methods to solve these three problems correctly. Thus, the researcher divided the students based on the problem-solving methods that they used, namely application of only one methods, correct application of two methods, and incorrect application of two methods, and summarized the results in Table 2.

As can be seen, half of the high prior knowledge students in the pretraining group could correctly apply two different methods to solve the three near transfer of learning problems, whereas less than a fourth of the high prior knowledge students in the segmented learning group could do so. Most of the low prior knowledge students in the two strategy groups could only apply one problem-solving method. Compared to the previous inferences, only the high prior knowledge students in the pretraining group perceived cognitive load within their cognitive capacities and were able the understand the two different problem-solving methods presented in the learning materials. The researcher speculates that this may be because the high prior knowledge students did not possess the two problemsolving sub-concepts in the materials, namely the drawing and interpretation of line segment diagrams. Thus, the element interactivity that they encountered while reading the segmented learning materials was still too high; aside from processing the information in the two steps, they also needed to process the integration of the two steps to solve the problem. This high element interactivity thus exceeded the cognitive capacity of the students. In contrast, the first part of the pretraining materials enabled students to focus on processing a single concept, and after reading two pairs of problems, most of the high prior knowledge students may already have had the sub-concept. When they subsequently read the explanation on how to apply the sub-concept to solve problems, they had already formed the sub-schema, which reduced the amount of element interactivity in the information. This enabled them to more successfully understand the content of the materials without exceeding their cognitive capacity and achieving better learning effectiveness.

For the low prior knowledge students, even though the pretraining materials enabled them to focus on processing a sub-concept, the provision of only two pairs of problems, one with an unknown reference quantity and the other with an unknown comparison quantity, was probably still not enough practice for them to establish the sub-concept. As for the students in the segmented learning group, the element interactivity was too high for even the high prior knowledge students, let alone the low prior knowledge students. 


\section{Implications for Instruction}

\section{Instruction design for effective intrinsic load management}

Previous research shows that the unit regarding reference quantities and comparison quantities in the sixthgrade mathematics curriculum is problematic for many sixth-graders in Taiwan. This study designed two sets of materials to manage intrinsic cognitive load. It is hoped that instruction design can be viewed in a systematic manner to provide elementary school teachers with useful reference.

\section{Appropriate instructional strategies based on learners' prior knowledge}

Although the instructional materials designed to manage intrinsic load in past studies have all succeeded in enhancing the learning effectiveness of learners, the results of this study indicate that for most older elementary school students who are accustomed to using a single skill or concept to solve problems, pretraining is probably a more suitable instructional strategy to manage intrinsic load. If teachers do not clearly understand what prior knowledge is necessary, segmented learning is a more difficult strategy to implement.

\section{ACKNOWLEDGEMENTS}

This research was partially supported by Ministry of Science and Technology, Taiwan, R.O.C., under contract no. MOST 105-2410-H-142-007-MY2.

\section{REFERENCES}

Ayres, P. (2006a). Impact of reducing intrinsic cognitive load on learning in a mathematics domain. Applying cognitive psychology, 20, 287-298. https://doi.org/10.1002/acp.1245

Ayres, P. (2006b). Using subjective measures to detect variations of intrinsic load within problems. Learning and Instruction, 16, 389-400. https:/ / doi.org/10.1016/j.learninstruc.2006.09.001

Ayres, P. (2013). Can the isolated-elements strategy be improved by targeting points of high cognitive load for additional practice? Learning and Instruction, 23, 115-124. https:/ / doi.org/10.1016/j.learninstruc.2012.08.002

Brünken, R., Seufert, T., \& Paas, P., (2010). Measuring cognitive load. In J. L. Plass, R. Moreno, and R. Brünken (Eds.), Cognitive load theory and research in educational psychology (pp. 181-202). New York, NY: Cambridge University Press. https://doi.org/10.1007/978-1-4419-8126-4_6

Clarke, T., Ayres, P., \& Sweller, J. (2005). The impact of sequencing and prior knowledge on learning mathematics through spreadsheet applications. Educational Technology, Research and Development, 53, 15-24. https://doi.org/10.1007/BF02504794

DeLeeuw, K. E., \& Mayer, R. E. (2008). A comparison of three measures of cognitive load: evidence for separable measures of intrinsic, extraneous, and germane load. Journal of Educational Psychology, 100, 223-234. https:/ / doi.org/10.1037/0022-0663.100.1.223

Galy, E., Cariou, M., \& Mélan, C. (2012). What is the relationship between mental workload factors and cognitive load types? International Journal of Psychophysiology, 83, 269-275. https://doi.org/10.1016/j.ijpsycho.2011.09.023

Huang J. -C (Ed.) (2012). Elementary Mathematics Textbook, 12, Tainan, Taiwan, Nani Press.

Huang, Y.-H., \& Shie J.-T (2016). The Comparison of Two Mathematics Problem-Solving Strategies of Line-Diagram Representations on Learning Achievements. Bulletin of Educational Psychology, 47(4), 581-601. https:// doi.org/10.6251/BEP.20150603

Kalyuga S. (2015). Instructional guidance: A cognitive load perspective, Charlotte, NC, USA: Information Age Publishing.

Kester, L., Kirschner, P. A., \& van Merriënboer, J. J. G. (2004a). Information presentation and trouble -shooting in electrical circuits. International Journal of Science Education, 26(2/6), 239-256. https:/ / doi.org/10.1080/69032000072809

Kester, L., Kirschner, P. A., \& van Merriënboer, J. J. G. (2004b). Just in time presentation of different types of information in learning statistics. Instructional Science, 32, 233-252. https:/ / doi.org/10.1023/B:TRUC.0000024191.27560.e3

Kester, L., Kirschner, P. A., \& van Merriënboer, J. J. G. (2006). Just-in-time information presentation: Improving learning and troubleshooting skill. Contemporary Educational Psychology, 31, 167-185. https:// doi.org/10.1016/j.cedpsych.2005.04.002 
Kester, L., Paas, P., \& van Merriënboer, J. J. G. (2010). Instructional control of Cognitive Load in the Design of Complex Learning Environments., In J. L. Plass, R. Moreno, \& R., Brünken (Eds.), Cognitive load theory and research in educational psychology (pp. 109-130). New York, NY: Cambridge University Press.

Lee, Y.-C. (2013). A Study of Line Segment Diagram Representation on Reference Quantity Problem Solving Performance for Sixth Grader (Unpublished master's thesis), National Pingtung University of Education, Pingtung, Taiwan.

Leppink, J., \& van den Heuvel, A. (2015). The evolution of cognitive load theory and its application to medical education. Perspectives on Medical Education, 4, 119-127. https://doi.org/10.1007/s40037-015-0192-x

Leppink, J., Paas, F., van der Vleuten, C. P. M., Van Gog, T., \& van Merriënboer, J. J. G. (2013). Development of an instrument for measuring different types of cognitive load. Behavior Research Methods, 45(4), 1058-1072. https:/ / doi.org/10.3758/s13428-013-0334-1

Leppink, J., Paas, F., van Gog, T., van der Vleuten, C. P. M., \& van Merriënboer, J. J. G. (2014). Effects of pairs of problems and examples on task performance and different types of cognitive load. Learning and Instruction, 30, 32-42. https:/ / doi.org/10.1016/j.learninstruc.2013.12.001

Mayer, R. E., \& Moreno, R. (2003). Nineways to reduce cognitive load in multimedia learning. Educational Psychologist, 38, 43-52. https://doi.org/10.1207/S15326985EP3801_6

Mayer, R., \& Moreno, R. E. (2010). Techniques that reduce extraneous cognitive load and manage intrinsic cognitive load during multimedia learning. In J. L. Plass, R. Moreno, \& R. Brünken (Eds.), Cognitive load theory and research in educational psychology. (pp. 131-152). New York: Cambridge University Press. https:/ / doi.org/10.1017/CBO9780511844744.009

Pollock, E., Chandler, P., \& Sweller, J. (2002). Assimilating complex information. Learning and Instruction, 12, 61-86. https:/ / doi.org/10.1016/S0959-4752(01)00016-0

Renkl, A., \& Atkinson, R. K. (2010). Learning from worked-out examples and problem solving. In J. Plass, R. Moreno, \& R. Brünken (Eds.), Cognitive load theory and research in educational psychology (pp. 91-108). New York: Cambridge University Press. https:/ / doi.org/10.1017/CBO9780511844744.007

Sweller, J. (1988). Cognitive load during problem solving: effects on learning. Cognitive Science, 12, 257-285. https://doi.org/10.1207/s15516709cog1202_4

Sweller, J. (2010a). Element interactivity and intrinsic, extraneous, and germane cognitive load. Educational Psychology Review, 22, 123-138. https:/ / doi.org/10.1007/s10648-010-9128-5

Sweller, J. (2010b). Cognitive load theory: recent theoretical advances. In J. L. Plass, R. Moreno, and R. Brünken (Eds.), Cognitive load theory and research in educational psychology (pp. 29-47). New York, NY: Cambridge University Press. https:/ / doi.org/10.1017/CBO9780511844744.004

Sweller, J., Ayres, P., \& Kalyuga S. (2011) Altering Element Interactivity and Intrinsic Cognitive load, Cognitive load theory (pp. 203-218). New York, NY: Springer-Verlag.

Wong, A., Leahy, W., Marcus, N., \& Sweller, J. (2012). Cognitive load theory: The transient information effect and e-learning. Learning and Instruction, 22, 449-457. https:/ / doi.org/10.1016/j.learninstruc.2012.05.004

\section{http://www.ejmste.com}

